\title{
Exploring somatization types among patients in Indonesia: latent class analysis using the Adult Symptom Inventory
}

\author{
Wahyu Widhiarso $^{A, B, C, D, E, F}$, M. Noor Rochman Hadjam ${ }^{A, B, E}$ \\ Faculty of Psychology, Universitas Gadjah Mada, Bulaksumur, Yogyakarta, Daerah Istimewa Yogyakarta, Indonesia
}

\section{BACKGROUND}

The aim of this study was to explore somatization types by reducing patient complaints to their most basic and parsimonious characteristics. We hypothesized that there were latent groups representing distinct types of somatization.

\section{PARTICIPANTS AND PROCEDURE}

Data were collected from patients undergoing both inpatient and outpatient treatment at two hospitals in Yogyakarta, Indonesia $(N=212)$.

\section{RESULTS}

Results from latent class analysis revealed four classes of somatization: two classes (Classes 1 and 2) referring to levels of somatization and two classes (Classes 3 and 4) referring to unique types of somatization. The first two classes (Classes 1 and 2; low and high levels of somatization, respectively) corresponded to the number of different symptoms that patients reported out of the list of physical symptoms in the Adult Symptom Inventory. The second two classes (Classes 3 and 4; non-serious and critical complaints, respectively) corresponded to two different sets of symptoms. Patients in Class 3 tended to report temporary mild complaints that are common in daily life, such as dizziness, nausea, and stomach pain. Patients in Class 4 tended to report severe complaints and medical problems that require serious treatment or medication, such as deafness or blindness.

\section{CONCLUSIONS}

The present study do confirm somatization as a unidimensional experience reflecting a general tendency to report somatic symptoms, but rather support the understanding of somatization as a multidimensional construct.

\section{KEY WORDS}

physical complaints; type of somatization; latent class analysis

CORRESPONDING AUthor - Wahyu Widhiarso, MA, Faculty of Psychology, Universitas Gadjah Mada, Jl. Humaniora No. 1, Yogyakarta 55281, Indonesia, e-mail: wahyu_psy@ugm.ac.id

aUthors' CONTRIBUtion - A: Study design - B: Data collection · C: Statistical analysis · D: Data interpretation . E: Manuscript preparation · F: Literature search · G: Funds collection

TO CITE THIS ARTICLE - Widhiarso, W., \& Hadjam, M. N. R. (2014). Exploring somatization type among patients in Indonesia: latent class analysis using the Adult Symptom Inventory. Current Issues in Personality Psychology, 2(4), 208-216. RECEIVED 24.09.2014 · REVIEWED 24.10.2014 · ACCEPTED 02.12.2014 · PUBLISHED 19.12.2014 


\section{BACKGROUND}

Somatization disorder is a type of physical complaint that interrupts health and affects performance. This disorder refers to a specific physical ailment or complaint with no medical evidence. Somatization, according to Ford (1983), is a process that involves the physical (soma) for psychological or personal reasons. All of the physical complaints of somatization have specific causes, such as internal or interpersonal conflicts, or social or environmental problems. Lipowski (cited in Mayou, 1993) states that somatic disorder is a tendency to communicate an unpleasant psychological condition in the form of physical complaints and symptoms, which tend to elicit medical help. Somatization is a form of somatoform disorder. The source of the disorder is anxiety, which is manifested in physical complaints; were it not for these physical complaints, others would be unaware that the person was suffering from anxiety (Davison $\&$ Neale, 2001). Somatization has no clear medical explanation. This disorder was originally known as Briquet's syndrome, after Paul Briquet, who first identified patients who complained of medical symptoms without any evidence (Mayou, 1993). Kaplan and Sadock (1991) explain somatic disorder as a chronic physical disorder that cannot be explained medically, and which is related to psychological tensions and problems.

A person with somatization not only complains of physical ailments, but also wants to obtain medical help and treatment (Barsky, 1992). Somatization disorder is a psychological response manifested through the body (soma) for psychological or personal purposes (Ford, 1983), and has the same symptoms as psychosomatic disorders and hypochondriasis, with all three being manifested through physical complaints. The only difference is that psychosomatic disorder involves actual physical illness and medical evidence, as it is rooted in genuinely distressing chronic psychological problems. These problems can disrupt the sympathetic nervous system and biochemical system, affecting various organs and the hormonal system. Hypochondriasis refers, instead, to an overwhelming anxiety about one's health. A person with hypochondriasis is always haunted by the perception that he or she suffers from a serious and specific disease. Persons with hypochondriasis complain that their body has certain specific defects or problems, and express unrealistic fears, worries, and anxiety. Real physical complaints in cases of hypochondriasis are very limited and specific.

\section{TYPES OF SOMATIZATION}

Somatization is a form of individual expression that uses pain as the complaint. Somatizing patients com- municate their emotions at the somatic level. As such, patients only report the specific pains to their physicians that those patients deem (at a subconscious level) relevant to communicating their distress; they do not report pains that are not relevant to their purpose. Obviously, the pain they have reported only includes the specific pain that according to them is relevant to be communicated. For somatization to be measured using self-report assessments, a number of specific pains must be categorized. For example, the Adult Somatization Inventory (ASI) contains 35 items regarding the types of pain or physical symptoms, while the Symptom Checklist-90-Revised Scale consists of 90 items (Derogatis, 1994). A person with somatization will not endorse all items in the measure, as feeling pain throughout the entire body is not common, and would not serve the patient's goal of communicating their psychic distress through rational, acceptable physical complaints.

Hence, it is only reasonable to expect patients to only report a few types of pain, which often correspond with what has been found in previous research. For example, Escobar, Burnam, Karno, Forsythe, and Golding (1987) proposed one type of somatization, abridged somatization, that can be used to refer to men or women experiencing four or more of six unexplained physical symptoms. Kroenke et al. (1997) proposed another type of somatization, multisomatoform disorder, to describe patients currently experiencing at least three unexplained physical symptoms. This research indicates that persons experiencing somatization should not report too many different physical symptoms. For this reason, exploring the different types of somatization in the patient population is an important goal of research.

According to Rosen, Kleinman, and Katon (1982), there are three types of somatization. The first type is common somatization, which occurs when patients present with somatic complaints after stressful events. This type is usually psycho-physiological in origin, and always manifests as headaches, epigastric distress, muscle spasms, and insomnia. These complaints may indicate stress-related autonomic nervous system arousal. The second type is sub-acute somatization, which occurs when patients with depression or panic disorders selectively focus on the somatic components of these illnesses. Complaints such as headaches, dizziness, and chest pain usually indicate this type. The third and final type is chronic somatization. This type frequently occurs in patients who have experienced chronic psychological pain such as being abandoned, physically abused, sexually abused, or severely neglected by their family.

Kirmayer and Robbins (1991) propose three alternative types of somatization, which represent three conceptually distinct patterns of experiencing and reporting somatic distress. The first type, functional somatization, is characterized by medically 
unexplained symptoms occurring in multiple physiological systems. In its most extreme manifestation, somatization disorder or undifferentiated somatoform disorder, this type is characterized by multiple types of chronic symptoms. The second form of somatization, hypochondriasis, entails worrying about a real or imagined illness beyond what is expected for the reported physical symptoms. The third type, exclusively somatic clinical presentation, occurs in individuals diagnosed with one or more mental disorders that do not fall under the somatoform disorder category.

Another type of somatization disorder is conversion disorder. Common symptoms include impaired coordination or balance, paralysis or localized weakness, difficulty swallowing or lump in throat, aphonia (inability to speak) urinary retention, hallucinations, loss of touch or the ability to feel pain, double vision, blindness, deafness, seizures, dissociative symptoms such as amnesia, and loss of consciousness other than fainting. The term conversion refers to the theory that unpleasant affect and conflicts that cannot be solved are somehow transformed into the reported somatic symptoms (World Health Organization, 1992).

Based on the types described above, it appears that somatization can be categorized based on certain types. The types of somatization presented above were developed based on both theory and the intensive study of many patients experiencing somatization. The present study explores these types of somatization using contemporary methods, which differ from those methods previously used. In this study, the types of somatization were explored using latent class analysis (LCA). Prior exploration of somatization types has been done by Kato (Kato, Sullivan, \& Pedersen, 2010), who found a five-class solution. Kato suggests redefining the existing classification criteria for functional somatic syndromes in terms of a single (uncomplicated) or multiple (complicated) syndromes. Our study replicates Kato's study using different measurement instruments, and using a sample of patients in Indonesia.

\section{LATENT CLASS ANALYSIS}

In the previous research, the mapping of patient types was conducted using a top-down theory-driven approach (Duhe, 2009). In contrast, the current study employs a new analysis method for mapping patient types. Recent analysis developments show that exploring types of individuals using the bottom up approach (i.e., using individual response patterns to the measure) is possible (Eid \& Zickar, 2007). Because the type of individuals has not been previously determined, this type is called a latent class. Hence, this technique is called latent class analysis (LCA; Nylund, Asparouhov, \& Muthén, 2007). The purpose of this technique is to find groups of individuals based on its members' response pattern on certain measures. Just as exploratory factor analysis reveals latent factors derived from a set of items, LCA divides respondents into groups. While exploratory factor analysis maps the items that measure similar attributes, LCA aims to map the respondents by their response patterns. The difference between LCA and factor analysis is the object of analysis: the unit analysis of factor analysis is a set of items, while the unit analysis of LCA is a set of individuals.

The present study aims to explore the categories of somatization based on individual responses to a somatization inventory. This study is based on the assumption that somatization is a complex psychological construct. The complexity of somatization means that common instruments can only assess the relative severity of symptoms, but cannot divide patients into different somatization types. The obtained score therefore only permits ordinal (i.e. severity) categorization but not nominal (i.e. type) categorization. Ordinal categorization indicates ranking between categories, and nominal categorization indicates no ranking between categories (Tokuda et al., 2009).

\section{AIM OF THE STUDY}

In the present study, we analyzed 35 medical complaints that commonly appear as somatic symptoms. These symptoms included abdominal discomfort and gastro-esophageal reflux, general muscular pain, fatigue, dizziness, autonomic dysfunction, breathlessness, back pain, and recurrent headache. To cluster these symptoms without assuming any existing categorization, we employed LCA to empirically test the existence of discrete groups with similar patterns of symptoms.

\section{PARTICIPANTS AND PROCEDURE}

\section{PARTICIPANTS}

Participants were individuals undergoing either inpatient or outpatient treatment at PKU Muhammadiyah and Bethesda Hospital in Yogyakarta, Indonesia $(N=212)$. These hospitals were selected based on technical considerations (i.e., their availability to the researchers). Prospective participants were selected by a psychologist who works in both hospitals, and who used observation and interviews over a period of several months to identify patients who reported notable physical complaints without medical evidence. Participants having diverse backgrounds were selected; however, $73 \%$ were female. This was expected, as based on the results of previous research, women are more prone to somatization (Barsky, Peekna, 
\& Borus, 2001). The average age of the participants was 55.40 years $(S D=11.00)$; age groups were late adolescence (18-24 years old; 43\%), adults (25-60 years old; $51 \%$ ), elderly (> 60 years old; $2 \%$ ) and not identified (5\%). Based on marital status, participants can be divided into three categories: widow/widower (1\%), married (27\%), not yet married (72\%). Sixty-two percent of the participants were in either full- or part-time paid employment, $10 \%$ civil servants, $8 \%$ military/police, 3\% entrepreneurs, $1 \%$ teachers, $70 \%$ students, $1 \%$ farmers, and $6 \%$ unidentified. Based on educational background, there are four categories: elementary school (4\%), junior high school (10\%), senior high school (83\%), and undergraduate $(0.02 \%)$. Participants in the present study all experienced somatization and lived in Yogyakarta.

\section{INSTRUMENT}

The Adult Somatization Inventory (ASI; Garber, Walker, \& Zeman, 1991; Walker, Beck, Garber, \& Lambert, 2009) assesses the severity of nonspecific somatic symptoms. The ASI includes symptoms from the revised third edition of the Diagnostic and Statistical Manual of Mental Disorders (DSM-III-R), as well as from the somatization factor of the Hopkins Symptom Checklist. Respondents are asked to rate the extent to which they have experienced each of the 35 symptoms listed (such as "headaches", "feeling low in energy", or "faintness or dizziness") in the preceding two weeks on a 5-point Likert-type scale ranging from "not at all" (0) to "a whole lot" (4). Total score on the ASI can range from 0 to 140 . The three-month test-retest Pearson reliability for this instrument was reported to be .50 for healthy controls and .66 for patients experiencing chronic pain (Garber et al., 1991). The ASI has four factors - pseudo-neurological, cardiovascular, gastrointestinal, and pain/weakness which correspond to the categories of somatic complaints in the diagnostic classification of somatoform disorder.

\section{ANALYSES}

The present study employed LCA to identify groups of patients based on their responses to the somatization inventory. These groups are referred to as latent classes because they are not directly observed, but rather inferred based on the results of the analysis. The optimal number of classes is decided by analyzing several models with an increasing number of classes, and then comparing goodness of fit with the study findings. Models were fitted using maximum likelihood estimation with robust standard errors. Classification quality was assessed using the recommended indices, including the Akaike information criterion (AIC), Bayesian information criterion (BIC), and the normalized entropy criterion. Each of these types of criteria can be interpreted in different ways. Information criterion values (AIC and BIC) are used to choose the best model among competing models. Theoretically, lower AIC and BIC criterion values indicate a better model. However, there is no rule that indicates the cut-off value of AIC and BIC. The selected model is determined by the decreasing value of AIC and BIC between models with smaller and larger number of classes. The best-fitting model is determined if the decrease of AIC and BIC values is small. There are no set cut-off criteria for deciding whether the entropy is reasonably high, but an entropy value near 1.00 suggests a reasonable model fit (Jung $\&$ Wickrama, 2008). Missing data were excluded from the analysis using listwise deletion. Latent class analyses were performed using MPLUS 7.0.

\section{RESULTS}

The purpose of this study was to explore the types of somatic disorder by reducing indirect indicators of this disorder to more basic indicators. We hypothesized that there were latent groups, associated with their type of somatization, based on reported physical complaints. Latent class analysis allowed us to test this hypothesis by exploring any possible classes or segments of participants.

Table 1 reflects the goodness-of-fit statistics for the proposed models applied to the study sample. Based on the comparison of statistical criteria, the model with four latent classes fits the data best. To select the model that had the best fit, was most interpretable, and was most parsimonious, we specifically examined the BIC and the AIC across the proposed models. The BIC and AIC are widely accepted for LCA methods, although the BIC performs slightly better than the AIC (Lanza, Collins, Lemmon, \& Schafer, 2007; Yang, 2006).

The analysis showed that the values for the AIC, BIC, and the Adjusted BIC substantially declined from a one-class (e.g., AIC $=9078.10$ ) to a three-class model (e.g., AIC = 6585.17). However, a substantial decrease in the values of the AIC and the BIC did not occur between four-class (e.g., AIC = 6147.16) and five-class models (e.g., AIC $=6042.35$ ). For this reason, the four-class model can be assumed to explain the data better than the previous classes of models.

We checked whether a five-class model might explain the data even better than a four-class model. The decrease in AIC values between the four-class and five-class model was quite large, but this finding was not repeated in the BIC values, as moving from a four-class to a five-class model resulted in an increased BIC value. Further, the four-class model was more interpretable and parsimonious than the 
Exploring somatization types

Table 1

Class membership percentage and the description of each class based on item endorsement

\begin{tabular}{|c|c|c|c|}
\hline Classes & Membership & Name & Information \\
\hline Class 1 & $40.25 \%$ & Low level of somatization & Low probability for all items \\
\hline Class 2 & $16.58 \%$ & High level of somatization & High probability for all items \\
\hline Class 3 & $21.07 \%$ & $\begin{array}{l}\text { Non-serious pain that can } \\
\text { daily occur }\end{array}$ & $\begin{array}{c}\text { Faintness/dizziness, nausea, stomach pain, } \\
\text { headache, heart or chest pain, } \\
\text { Low energy, sore muscles, } \\
\text { hot or cold spells, weakness }\end{array}$ \\
\hline Class 4 & $22.08 \%$ & Critical pain or suffering & $\begin{array}{l}\text { Trouble breathing, blurred vision, } \\
\text { Deafness, blindness, fainting, amnesia, seizures, } \\
\text { muscle weakness, difficulty urinating }\end{array}$ \\
\hline
\end{tabular}

Table 2

Goodness-of-fit indicator values for each model class

\begin{tabular}{cccccc}
\hline & Class 1 & Class 2 & Class 3 & Class 4 & Class 5 \\
\hline AIC & 9078.10 & 7122.36 & 6585.17 & 6147.16 & 6042.35 \\
BIC & 9195.58 & 7360.68 & 6944.32 & 6627.15 & 6643.18 \\
Adjusted BIC & 9084.68 & 7135.70 & 6605.28 & 6174.03 & 6075.99 \\
Entropy & - & 0.99 & 0.97 & 0.98 & 0.98 \\
\hline
\end{tabular}

five-class model; hence, we concluded that the fourclass model better explained the study sample than the other models. The entropy value, which indicates the accuracy of the individual fit for inclusion in existing classes, had satisfactory results, with values above .90 . The comparison of goodness-of-fit indices of each is given in Table 2.

Comparing among the one-, two-, three- and four-class models, the distribution of respondents based on patterns in their responses to the ASI is best served by a model with four classes. In models with three classes, BIC values decreased quite substantially when the model changed from one-class to two-class, and from two-class to three-class, but the decrease was not sufficient when the model changed from three-class to four-class (see Table 1). This pattern also occurred for AIC values.

The largest numbers of study participants (40.25\%) were categorized in Class 1 . This class consisted of respondents with low-level somatization, who had a consistently low probability (on average, 4\%) of reporting any of the physical symptoms measured by the ASI. Unlike Class 1, Class $2(16.58 \%)$ consisted of participants with consistently high levels of somatization. On average, they had an $82 \%$ probability of reporting any somatization symptoms. The relationship between Classes 1 and 2 is ordinal, as the categories do not merely denote different somatization, but different levels of severity. The profile of each class is given in Figure 1.

Class 3 showed a relatively high prevalence of faintness, dizziness, nausea, stomach pain, headache, heart or chest pain, low energy, sore muscles, hot or cold spells, and weakness. Participants reported symptoms of mild complaints, of types that are common in daily life and that can occur temporarily. For example, patients reported feeling faintness or dizziness at times, which suddenly disappeared without needing treatment or medication. This class was named the non-serious complaints class. Those patients assigned to Class 3 had the lowest probability of reporting almost any type of permanent pain requiring major medications, such as amnesia, deafness, or blindness.

Class 4 consisted of patients with serious or critical complaints. Persons in this group reported severe symptoms, such as sensory problems (e.g., blurred vision, deafness, blindness), trouble getting breath, loss of consciousness (e.g. fainting), memory loss, seizures, muscle weakness, and difficulty urinating. Respondents assigned to Class 4 had a low probability of endorsing an item (below 10\%) or reporting any musculoskeletal problems in the limbs, such as pain in the arms or legs, hot or cold spells, pain in the lower back, or pain in the arms, knees, or elbows. 


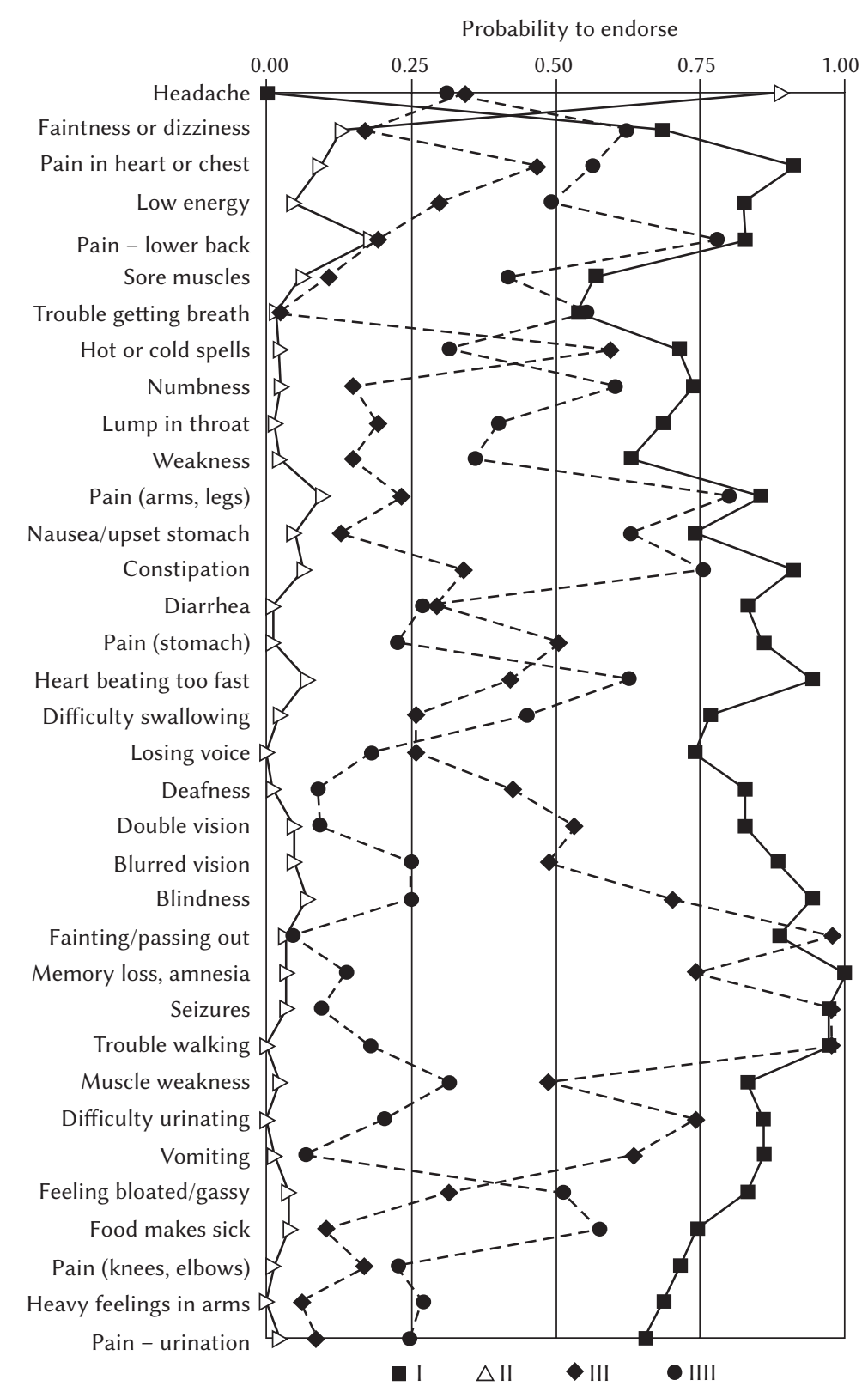

Figure 1. Profile of the four classes revealed by the present study.

Classes 3 and 4 were unique, in that members of Class 4 reported only a few pains listed on the ASI. The relationship between Classes 3 and 4 was horizontal, since both of them followed a nominal categorization. This means that Classes 3 and 4 indicated the type of somatization experienced by study participants.

\section{DISCUSSION}

This study identified four classes of somatic disorder among patients: two of these classes (Classes 1 and 2) referred to the levels of somatization (low- and high-level somatization, respectively), while the other two classes (Classes 3 and 4) refer to unique types of somatization. Based on the response pattern to the
ASI, patients in Class 3 tended to report temporary, mild pains that are common in daily life (e.g., dizziness, nausea, stomach pain). In contrast, patients in Class 4 tended to report severe pain requiring serious treatment or medication (e.g., deafness, blindness).

The existence of two classes associated with the level of somatization implies that ASI items measure a single attribute (i.e. unidimensional). Thus, obtained scores can reflect a single patient attribute (i.e. somatization). The literature suggests that LCA can be used to identify the underlying scales for categorical indicators, allowing a set of items to be reduced to a single scale with a minimal loss of information (Skrondal \& Rabe-Hesketh, 2007). In the present study, the emergence of classes according to the level of somatization indicates that the ASI con- 
sists of homogeneous items, as a one-dimensional model would not fit the data if structural differences emerged among respondents. Structural differences are individual differences that exist among respondents, which affect their scores (Eid \& Zickar, 2007). In the case of the present study, LCA accommodates both structural differences between subpopulations and individual differences within subpopulations. These structural differences represent true differences between individuals; in the case of the present study, these structural differences refer to types of somatic disorder. Based on this finding from the LCA, we can conclude that the ASI can accurately assess individual differences for diverse types of somatization, because almost $60 \%$ of the study participants were accounted for in Classes 1 and 2.

The presence of Classes 3 and 4 indicates that there were two classes of patients in the study sample, with regard to symptom type. The summed scores for patients in Classes 3 and 4 do not indicate whether their level of somatization is mild, moderate, or severe. These patients have particular characteristics that cannot be explained by the ASI. For example, patients in Class 3 commonly report nausea, which is a common complaints. However, this does not necessarily indicate that those patients have low levels of somatization; conversely, patients in Class 4 commonly report symptoms such as blindness, a serious medical condition, but patients in this group do not automatically have high levels of somatization. Level of somatization is not indicated by membership in Class 3 or 4 , but instead by other variables, such as increased likelihood of seeking treatment from multiple providers (Katon, Berg, Robins, \& Risse, 1986).

Class 3 consists of patients who report only mild complaints. The existence of this class of patients with somatic disorders is supported by several studies that have presented groups of patients who present with progressive fatigue, associated with a variety of other loosely bound somatic symptoms; dizziness is common, as well as muscle pain (Trimble, 2004). Bernstein et al. (1997) found the most commonly reported somatic complaints to include headaches, low energy, sore muscles, nausea or upset stomach, back pain, stomach pain, blurred vision, weakness, and food intolerance, as were reported by patients in Class 3 of the present study. Additionally, Taylor, Szatmari, Boyle, and Offord (1996) found that over $45 \%$ of surveyed adolescents reported frequent headaches and stomachaches. As somatization has been well established as an emotionally "safe" way to communicate discomfort, we believe that patients in Class 3 are communicating their psychic distress using a set of non-severe physical complaints.

Class 4, in contrast, consisted of patients with serious or critical complaints, such as sensory problems (e.g., blurred vision, deafness, blindness). The types of symptoms reported by patients in this class are similar to the symptoms of conversion disorder, one type of somatoform disorder. Patients with conversion disorder have pseudo-neurological symptoms (Bruno, 2002). The blindness, amnesia, and blurred vision commonly reported by patients in Class 4 are typical of the symptoms reported by persons with conversion disorder.

Patients in Class 4 tended to report only a few symptoms, but those symptoms that they did report tended to be quite serious in nature. The number of patients in the study sample that fell into Class 4 was quite large $(22.08 \%)$ - this percentage is equivalent to the number of patients in Class 3, which was $21.07 \%$. This number suggests that the ASI has limited predictive power for patients with this type (Class 4) of somatization. Composite scores (summed scores for all scale items) of patients in Class 4 were only moderate, because they reported few mild physical symptoms. Their relatively moderate scores may indicate a low or a high level of somatization, but this classification requires additional information, such as information on the consistency, severity, or intensity of patient efforts to communicate their distress.

Understanding somatization types is essential to understanding what persons reporting somatization are trying to express. Somatization is a unique medical condition, in that patients are not passive recipients of diagnoses, but rather dynamic participants, as somatic complaints are often influenced by individual perceptions of their own illnesses (Dornelas, 2004). Thus, considering the different types of somatization as representing different ways that patients are trying to communicate their distress will facilitate a better understand of somatization. This approach does not primarily view somatization according to the specific types of symptoms, but rather by the intensity or depth of those symptoms. Indeed, a patient reporting only one or two symptoms may still be suffering from high levels of somatization.

The results of the present study indicate that the scores obtained from instruments such as the ASI, which ask about the presence or absence of a set of symptoms, do not fully categorize the level of somatization. There are a large number of individuals (43.15\%) with unique types of somatization, members of Class 3 and Class 4 . The large number of individuals in these two classes indicates that the ASI offers relatively little information about the general phenomenon of somatization. This result agrees with the findings from two previous studies (Liu, Clark, \& Eaton, 1997; Walker et al., 2009), both of which found that all items on the ASI loaded strongly on a stable general factor, although smaller factors also were evident. Evidently, measuring somatization using the ASI remains problematic; this problem of assessment needs to be resolved to better identify and treat individuals with unique types of somatization. One possible way that the existence of this unique type of somatization could be accommodated would 
be to split the scale according to what specific construct was being measured. For example, the subscale score based only on a set of items that measure a specific type of somatization could be used, as opposed to a full score.

The assessment of somatic disorders via creating total scores that sum the number of physical complaints falsely views somatization as a unidimensional illness. Creating subscales that sum responses pertaining to different symptom types, as defined by the criteria for the various forms of somatization disorders, is consistent with the view of somatization as a multidimensional construct (Walker et al., 2009). The findings of the present study do not present somatization as a unidimensional experience reflecting a general tendency to report somatic symptoms, but rather support the understanding of somatization as a multidimensional construct.

\section{REFERENCES}

Barsky, A. J. (1992). Amplification, somatization, and the somatoform disorders. Psychosomatics, 33, 2834. DOI: 10.1016/S0033-3182(92)72018-0.

Barsky, A. J., Peekna, H. M., \& Borus, J. F. (2001). Somatic symptom reporting in women and men. Journal of General Internal Medicine, 16, 266-275. DOI: 10.1046/j.1525-1497.2001.016004266.x.

Bernstein, G. A., Massie, E. D., Thuras, P. D., Perwien, A. R., Borchardt, C. M., \& Crosby, R. D. (1997). Somatic symptoms in anxious-depressed school refusers. Journal of the American Academy of Child and Adolescent Psychiatry, 36, 661-668.

Bruno, F. J. (2002). Psychology A Self-Teaching Guide. Hoboken, NJ: John Wiley \& Sons, Inc.

Davison, C. D., \& Neale, M. J. (2001). Abnormal Psychology. New York: John Wiley and Sons, Inc.

Derogatis, L. R. (1994). Symptom Checklist-90-Revised: administration, scoring and procedures manual. National Computer Systems, Inc.: Minneapolis.

Dornelas, E. A. (2004). Personality-guided therapy for treating medical patients. In: J. J. Magnavita (ed.), Handbook of Personality Disorders: Theory and Practice. Hoboken, NJ: John Wiley \& Sons, Inc.

Duhe, S. (2009). What's your type? Using the Myers-Briggs Personality Inventory to improve team performance. Communication Teacher, 23, 142-147. DOI: 10.1080/17404620903218775.

Eid, M., \& Zickar, M. J. (2007). Detecting response styles and faking in personality and organizational assessments by mixed Rasch models. In: M. Von Davier, \& C. Carstensen (eds.), Multivariate and Mixture Distribution Rasch Models. New York: Springer.

Escobar, J. I., Burnam, M., Karno, M., Forsythe, A., \& Golding, J. M. (1987). Somatization in the com- munity. Archives of General Psychiatry, 44, 713718. DOI: 10.1001/archpsyc.1987.01800200039006.

Ford, C. V. (1983). The Somatizing Disorder. Illness as A Way of Life. New York: Elsevier Science Publishing Co. Inc.

Garber, J., Walker, L. S., \& Zeman, J. (1991). Somatization symptoms in a community sample of children and adolescents: Further validation of the Children's Somatization Inventory [Empirical Study]. Psychological Assessment: A Journal of Consulting and Clinical Psychology, 3, 588-595. DOI: 10.1037/1040-3590.3.4.588.

Jung, T., \& Wickrama, K. A. S. (2008). An introduction to latent class growth analysis and growth mixture modeling. Social and Personality Psychology Compass, 2, 302-317. DOI: 10.1111/j.17519004.2007.00054.x.

Kaplan, H. I., \& Sadock, B. J. (1991). Synopsis of Psychiatry. Maryland: William and Wilkins.

Kato, K., Sullivan, P. F., \& Pedersen, N. L. (2010). Latent class analysis of functional somatic symptoms in a population-based sample of twins. Journal of Psychosomatic Research, 68, 447-453. DOI: http://dx.doi.org/10.1016/j.jpsychores.2010.01.010.

Katon, W., Berg, A., Robins, A., \& Risse, S. (1986). Depression: Pattern of medical utilization and somatization in primary care. In: S. McHugh, \& T. M. Vallis (eds.), Illness behavior: a multidisciplinary model. New York: Plenum Press.

Kirmayer, L. J., \& Robbins, J. M. (1991). Three forms of somatization in primary care: prevalence, co-occurrence, and sociodemographic characteristics. Journal of Nervous \& Mental Disease, 179, 647-655. DOI: 10.1097/00005053-199111000-00001.

Kroenke, K., Spitzer, R. L., deGruy III, F. V., Hahn, S. R., Linzer, M., Williams, J. B. W., Brody, D., \& Davies, M. (1997). Multisomatoform disorder: An alternative to undifferentiated somatoform disorder for the somatizing patient in primary care. Archives of General Psychiatry, 54, 352-358. DOI: 10.1001/archpsyc.1997.01830160080011.

Lanza, S. T., Collins, L. M., Lemmon, D. R., \& Schafer, J. L. (2007). PROC LCA: A SAS Procedure for Latent Class Analysis. Structural Equation Modeling: A Multidisciplinary Journal, 14, 671-694. DOI: 10.1080/10705510701575602.

Liu, G., Clark, M. R., \& Eaton, W. W. (1997). Structural factor analyses for medically unexplained somatic symptoms of somatization disorder in the Epidemiologic Catchment Area Study. Psychological Medicine, 27, 617-626. DOI: 10.1017/ S0033291797004844.

Mayou, R. (1993). Somatization. Psychotherapy and Psychosomatics, 59, 69-83. DOI: 10.1159/000288650.

Nylund, K. L., Asparouhov, T., \& Muthén, B. O. (2007). Deciding on the number of classes in latent class analysis and growth mixture modeling: a Monte Carlo simulation study. Structural Equation Mod- 
eling: A Multidisciplinary Journal, 14, 535-569. DOI: $10.1080 / 10705510701575396$.

Rosen, G., Kleinman, A., \& Katon, W. (1982). Somatization in family-practice - a biopsychosocial approach. Journal of Family Practice, 14, 493-502.

Skrondal, A., \& Rabe-Hesketh, S. (2007). Latent variable modelling: a survey. Scandinavian Journal of Statistics, 34, 712-745. DOI: 10.1111/j.14679469.2007.00573.x.

Taylor, D. C., Szatmari, P., Boyle, M. H., \& Offord, D. R. (1996). Somatization and the vocabulary of everyday bodily experiences and concerns: a community study of adolescents. Journal of the American Academy of Child \& Adolescent Psychiatry, 35, 491-499. DOI: http://dx.doi.org/10.1097/00004583-19960400000015.

Tokuda, Y., Okubo, T., Ohde, S., Jacobs, J., Takahashi, O., Omata, F., Yanai, H., Hinohara, S., \& Fukui, T. (2009). Assessing Items on the SF-8 Japanese version for health-related quality of life: a psychometric analysis based on the nominal categories model of item response theory. Value in Health, 12, 568-573. DOI: 10.1111/j.1524-4733.2008.00449.x.

Trimble, M. (2004). Somatoform Disorders: A Medicolegal Guide. Cambridge: Cambridge University Press.

Walker, L. S., Beck, J. E., Garber, J., \& Lambert, W. (2009). Childrens Somatization Inventory: Psychometric Properties of the Revised Form (CSI-24). Journal of Pediatric Psychology, 34, 430-440. DOI: 10.1093/jpepsy/jsn093.

World Health Organization. (1992). The ICD 10 Classification of Mental and Behavioural Disorders. Geneva: World Health Organization.

Yang, C.-C. (2006). Evaluating latent class analysis models in qualitative phenotype identification. Computational Statistics \& Data Analysis, 50, 1090-1104. DOI: http://dx.doi.org/10.1016/j. csda.2004.11.004. 